\title{
Design Optimization with Geometric Programming for Core Type Large Power Transformers
}

\author{
Tamás Orosz (Doctoral student, Budapest University of Technology and Economics), \\ István Vajda (Professor, Óbuda University)
}

\begin{abstract}
A good transformer design satisfies certain functions and requirements. We can satisfy these requirements by various designs. The aim of the manufacturers is to find the most economic choice within the limitations imposed by the constraint functions, which are the combination of the design parameters resulting in the lowest cost unit. One of the earliest application of the Geometric Programming [GP] is the optimization of power transformers. The GP formalism has two main advantages. First the formalism guarantees that the obtained solution is the global minimum. Second the new solution methods can solve even large-scale GPs extremely efficiently and reliably. The design optimization program seeks a minimum capitalized cost solution by optimally setting the transformer's geometrical and electrical parameters. The transformer's capitalized cost chosen for object function, because it takes into consideration the manufacturing and the operational costs. This paper considers the optimization for three winding, three phase, core-form power transformers. This paper presents the implemented transformer cost optimization model and the optimization results.
\end{abstract}

Keywords - Design Optimization; Heuristic Algorithms; Mathematical programming; Power Engineering Computing; Power transformers.

\section{INTRODUCTION}

A good transformer design satisfies certain functions and requirements such as transforming power from one voltage level to another without overheating or without damaging itself when a short-circuit current or a lightning strike occurs. We can satisfy these requirements by various designs. The aim of the manufacturers is to find the most economical choice within the limitations imposed by the constraint functions, which are the combination of the design parameters whose result is the lowest cost unit. This cost optimization falls into the most general category of the non-linear optimization methods. In this area, there are no algorithms or iteration schemes which guarantee that we found the global optimum [1], [2]. When we choose an optimization model, there is also the question of how much detail to include in the problem description. Although the goal is to find the lowest cost design, one might wish that the solution should provide sufficient information, so that an actual design could be produced with little additional work [1], [2] and [3].

The transformer design is a mixture of science and art. Also nowadays, it relies mainly on the designer's knowledge and experience [2]. As far back as the beginning of the 20th century the manufacturers started to research optimization methods. Early research in transformer design attempted to reduce much of this judgment with analytical formulas.
Countless design procedures and a wide range of applied mathematical models can be found in the literature [1]-[9]. The first procedures replaced the different winding systems with their copper filling factor, and the aim is these methods to ascertain the optimal winding-core ratio. The first computer program for transformer optimization made by P.A. Abetti et al, in the General Electric corporation's laboratory in 1953 [1], [2], [3] and [5]. This program gave back the design variables, which provided sufficient information for a designer who made a solution for an offer from these data, which satisfies all of the requirements. A good example of the wide range of implemented mathematical models, that Andersen [4] is made an optimizing routine named Monica based on Monte Carlo simulation. The use of the artificial intelligence techniques in power transformer design like neural networks [6], [7], [8] or genetic algorithms [5], [6], give good evidence, that transformer design optimization is an active research field nowadays.

The importance of GP is based on relatively recent developments in solution methods which can solve even largescale geometric programs extremely efficiently and reliably [9]-[12]. Moreover, a geometric program can be converted into a convex optimization problem implying that the computed optimal solution is global.

One of the first applications of the GP is the transformer optimization [9]. This paper shows an optimization model which extends of these classical two winding GP optimization methods [2], [7] and solved by CVXOPT [12] very quickly. The aim of this optimization model is not to revise the final design, just to give an accurate hint for the designer at the beginning of the offer preparation stage.

\section{GEOMETRIC PROGRAMMING}

The GP is a branch of the non-linear optimization problems given in the standard form [2], [9]-[12]:

$$
\begin{aligned}
\operatorname{minimize}\left\{f_{0}\right\} \quad \text { subject to: } \\
f_{i}(x) \leq 1, \quad i=1, \ldots m, \\
g_{j}(x)=1, \quad j=1, \ldots, o
\end{aligned}
$$

where $x=\left(x_{1}, x_{2}, \ldots x_{n}\right)$ is a vector containing the optimization variables, $f_{i}(x)$ is a posynomial constraint inequality, $g_{j}(x)$ is a monomial constraint equality function. All elements of $x$ must be positive. The monomial function $g(x)$ expressed as:

$$
g(x)=c_{g} \cdot x_{1}^{\alpha_{1}} \cdot x_{2}^{\alpha_{2}} \ldots \cdot x_{n}^{\alpha_{n}},
$$

where $c_{g} \in \mathfrak{R}, \alpha_{i} \in \mathfrak{R}$ and $c_{g}>0$. 
A polynomial function is a linear combination of monomials:

$$
f(x)=\sum_{k=1}^{K} c_{k} \cdot x_{1}^{\alpha_{1 k}} \cdot x_{2}^{\alpha_{2 k}} \ldots x_{n}^{\alpha_{n k}} .
$$

The main requirement for solving a GP efficiently is to convert it into a convex optimization problem [10], [11]. A GP problem is non-convex in their general form, but most of them can be transformed into convex optimization problems by a logarithmic change of variables as $y_{i}=e^{a_{i}}$, and a transformation of the objective and constraint functions:

$$
\begin{aligned}
\operatorname{minimize}\left\{\log \left(f_{0}\left(e^{y}\right)\right)\right\} \quad \text { subject to. } \\
\log \left(f_{i}\left(e^{y}\right)\right) \leq 0, \quad i=1, \ldots m . \\
\log \left(g_{j}\left(e^{y}\right)\right)=0, \quad j=1, \ldots, o
\end{aligned}
$$

If this is done, convex optimization problem solvers can be used that are based on the efficient interior-point solving methods. The GP modeler does not need to know how GPs are solved; the transformation to a convex problem is handled by the applied solver. An open source implementation of the above mentioned primal-dual interior-point method is available in the CVXOPT [15] Python module.
The Optimization Model For Core-Form Power Transformers

The aim of the presented core-form transformer model is to give a sufficient solution for the cost optimization problem for offers. From the result parameters, the designer can make a solution with little additional work which satisfies all the mechanical, thermal and electrical constraints that are required for sophisticated design codes. This optimization model is mainly based on [2].

\section{A. Objective function}

The object function is the transformer capitalized cost. This consists of the manufacturing cost and the cost of the losses [13], [14] and can be expressed as

$$
C=A P_{n l l}+B P_{l l}+\sum_{k=0}^{n} C_{k} M_{k} .
$$

In this formula:

$A$ is the no-load loss capitalization factor in $€ / \mathrm{Kw}$,

$B$ is the load loss capitalization factor in $€ / \mathrm{kW}$,

$P_{l l}$ is the load loss of the transformer in $\mathrm{kW}$,

$P_{n l l}$ is the no load loss of the transformer in $\mathrm{kW}$,

$C_{k}$ is the sum of the unit manufacturing cost and the material cost of the transformer part in $€ / \mathrm{kg}$,

$M_{k}$ is the mass of the transformer part in $\mathrm{kg}$.

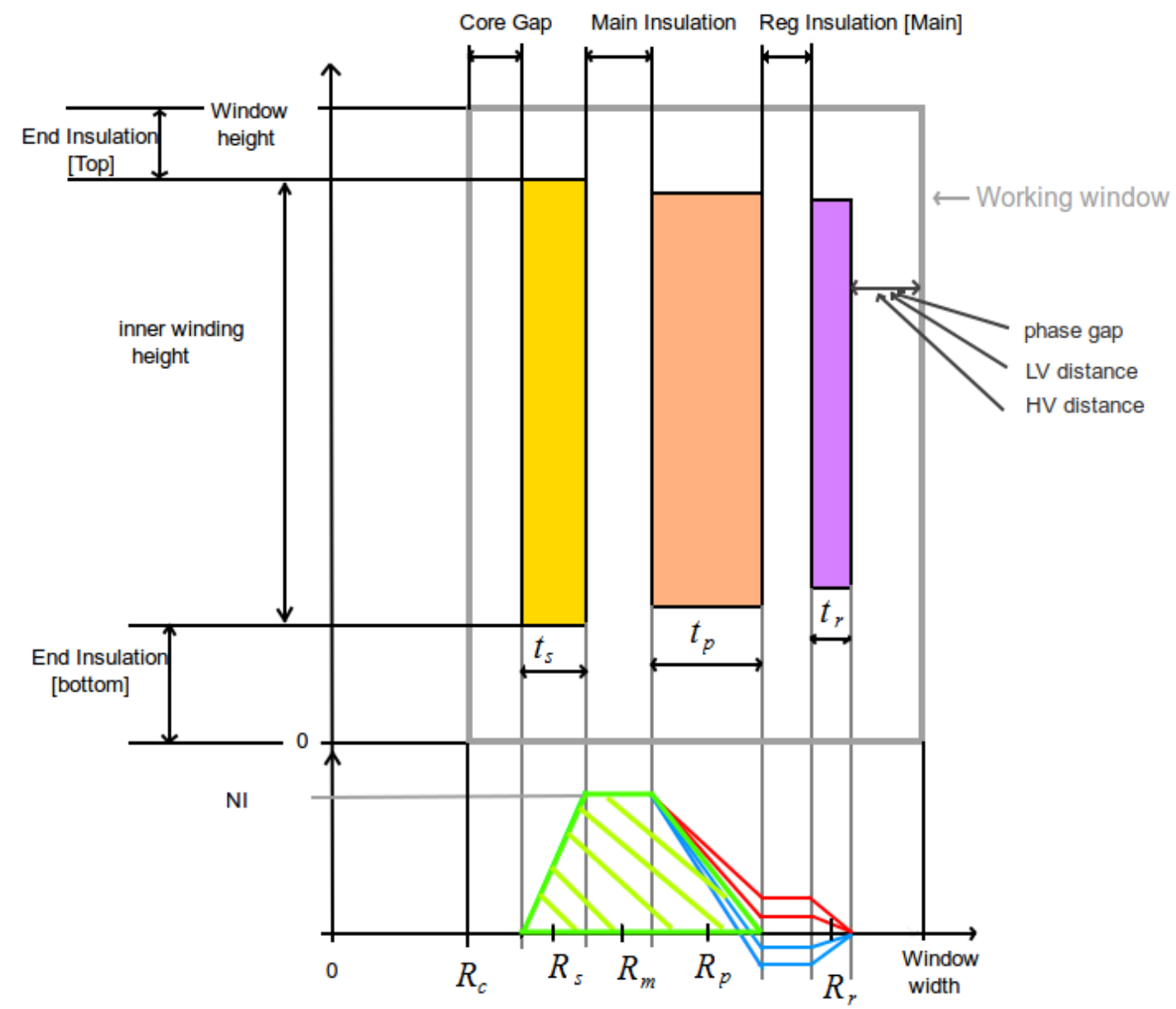

Fig. 1. Schematic view of the applied electrical and geometrical parameters in the transformer "Working window" model. 
In the optimization we take into consideration the active part of the transformer and the mass of the transformer tank. The transformer's active part consists of the core, the two main windings and the regulating winding in the presented model. These cost components are expressed with a basic set of design variables.

\section{B. Design variables}

The basic design variables in this optimization method are:

$P_{l l}$ is the load loss of the transformer in $\mathrm{kW}$,

$P_{n l l}$ is the no load loss of the transformer $\mathrm{kW}$,

$R_{s}$ is the mean radius of the secondary coil in $\mathrm{mm}$,

$t_{s}$ is the thickness of the secondary coil $\mathrm{mm}$,

$J_{s}$ is the current density of the secondary coil in $\mathrm{A} / \mathrm{mm}^{2}$,

$h_{s}$ is the height of the secondary coil in $\mathrm{mm}$,

$R_{p}$ is the mean radius of the primary winding in $\mathrm{mm}$,

$t_{p}$ is the thickness of the primary winding in $\mathrm{mm}$,

$J_{p}$ is the current density of the primary winding in $\mathrm{A} / \mathrm{mm}^{2}$,

$g_{m}$ is the main insulation distance in $\mathrm{mm}$,

$s$ is the radial width of the winding system in $\mathrm{mm}$,

$t_{r}$ is the thickness of the regulating winding in $\mathrm{mm}$,

$R_{r}$ is the radius of the regulating winding in $\mathrm{mm}$,

$B$ is the magnetic flux density in the core in T,

$M_{c}$ is the mass of the core in $\mathrm{kg}$,

$Z$ is the short-circuit impedance in $\%$.

\section{Inequality constraints}

1) Load loss estimation in a winding

$$
P_{l l}=\sum_{i} 2 \pi \rho_{C u} \lambda_{i} R_{i} t_{i} \alpha_{i} h_{i}(1+\kappa Z),
$$

where

$\rho_{C u}$ is the copper resistance in $\Omega \cdot \mathrm{m}$ at $75^{\circ} \mathrm{C}$,

$i$ represents a winding,

$\lambda_{i}$ is the copper filling factor in the winding $i$. This factor is an input parameter of the program. The value of parameter is well approximated by the designer from the voltage level, short-circuit impedance and phase current values,

$\kappa$ is a stray loss estimation factor described in [15], this paper we using $\kappa=4$ for the calculations, in the case of unshielded transformers,

$\alpha_{i}$ is the height ratio of the primary - secondary and the regulating.

\section{2) No-load loss calculation}

$$
P_{n l l}=M_{C} \cdot f_{b} \cdot \sum_{j=0}^{5} a_{j} x^{j}
$$

where

$f_{b}$ is the building-factor, which is the ratio of the measured and the calculated core losses from the Epstein-curvature. The value of $f_{b}$ depends on the core manufacturing technology, we chose $f_{b}=1.2$ in our calculations, $\sum_{j=0}^{5} a_{j} x^{j}$ is the fitted posynomial function to the material

Epstein-curve, which provided by the manufacturer.

\section{3) Core mass calculation}

As we use the working window terminology for the transformer calculation, we can easily generalize the optimization model for different transformer types by multiplying the results with the number of working windows, and write the mass of the core in the next generalized form:

$$
M_{\text {core }}=M_{\text {column }}+M_{\text {yoke }}+M_{\text {corner }}+M_{\text {sl }} .
$$

where

$M_{\text {corner }}$ is the sum of the corners mass in $\mathrm{kg}$,

$M_{\text {column }}$ is the mass of the columns in $\mathrm{kg}$,

$M_{y o k e}$ is the sum of the yokes, without the corners mass in $\mathrm{kg}$, $M_{s l}$ is the mass of the side-legs in $\mathrm{kg}$.

It is possible to explicate these masses to the next closed forms:

$$
\begin{aligned}
& M_{\text {corner }}=R_{c}^{2} \cdot \pi \cdot \eta_{c} \cdot \rho_{f e} \cdot\left(c \cdot R_{c} \cdot \zeta^{2} \cdot \gamma\right), \\
& M_{\text {column }}=R_{c}^{2} \cdot \pi \cdot \eta_{c} \cdot \rho_{f e} \cdot\left(h+e i_{b}+e i_{t}\right), \\
& M_{y o k e}=R_{c}^{2} \cdot \pi \cdot \eta_{c} \cdot \rho_{f e} \cdot(s \cdot s n+m \cdot m n), \\
& M_{s l}=R_{c}^{2} \cdot \pi \cdot \eta_{c} \cdot \rho_{f e} \cdot p \cdot h \cdot\left(h+e i_{b}+e i_{t} \cdot m n\right) .
\end{aligned}
$$

where

$\rho_{\mathrm{Fe}}$ is the density of the core material in $\mathrm{kg} / \mathrm{m}^{3}$,

$\zeta$ is the ratio of the leg and the side leg,

$c$ number of the corners in the applied transformer core shape,

$\gamma$ is factor, which take into consideration the mass growth in the corners. In this paper we chosen $\gamma=1.025$,

$e i_{b}$ and $e i_{t}$ is the length of the end insulation in the bottom and the top region, for the calculation we need only the sum of them in $\mathrm{mm}$,

$\eta_{\mathrm{c}}$ is the core filling factor, which takes into consideration the lamination, cooling ducts, etc. in \%, $s n$ is the number of the winding widths, $m$ the distance between two phase in $\mathrm{mm}$, $m n$ is the number of $m$ dimensions in the core shape, $p$ is the number of side-legs.

4) Window width

$$
g_{\text {core }}+t_{s}+t_{p}+t_{r}+g+g_{\text {reg }} \leq s,
$$

where

$g_{\text {core }}$ is the distance between the core and the secondary winding.

$g_{\text {reg }}$ is the distance between the regulating winding and the primary winding.

The window width variable is introduced to simplify the equation system when we use it for different core types, or different positions of the regulating winding are applied. 


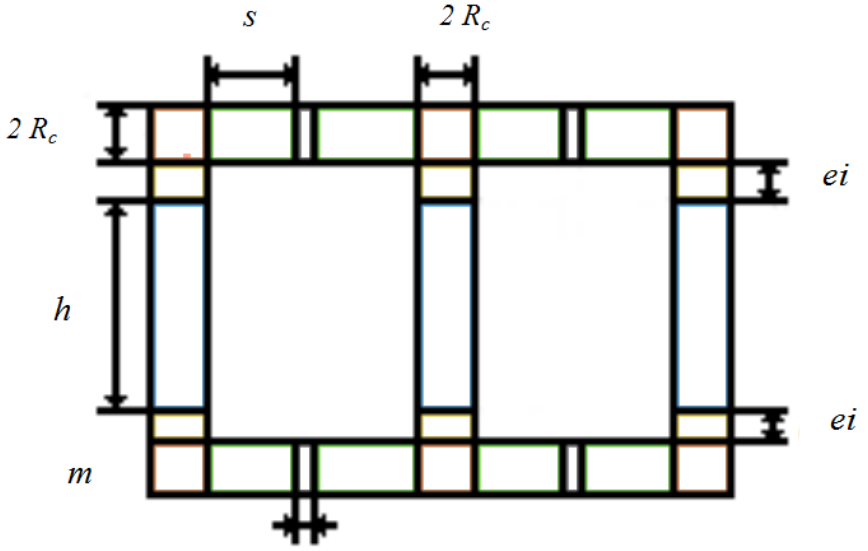

$m$

Fig. 2. Illustrates the different summarized parts in the case of common three phase, three legged transformer core. In this case $c=6, p=0$.

\section{5) Winding Arrangement}

Inequality to define $R_{s} \rightarrow R_{p}$ positions, which illustrated in the figure 1 is found as:

$$
R_{s}+g+\frac{t_{s}}{2}+\frac{t_{p}}{2} \leq R_{p} .
$$

Inequality to define the $R_{s} \rightarrow R_{c}$ positions:

$$
R_{c}+g_{\text {core }}+\frac{t_{s}}{2} \leq R_{s} .
$$

Inequality to define the $R_{p} \rightarrow R_{r}$ positions:

$$
R_{p}+g_{\text {reg }}+\frac{t_{p}}{2}+\frac{t_{r}}{2} \leq R_{r} .
$$

\section{6) Regulating Winding Dimensions}

The model assumes that we are using a diverter switch for the regulation and in the nominal state the regulating winding is de-energized. The regulating winding at that time reduces the short-circuit impedance of the transformer by their radial width

$$
\begin{gathered}
t_{r}=\frac{P_{r e g}}{j_{r e g}^{2} \cdot \alpha \cdot h_{s} \cdot U_{r e g} \cdot \lambda_{r e g}} \text { and } \\
P_{\text {reg }}=P_{\text {main }} \cdot \varepsilon_{\text {main }},
\end{gathered}
$$

where

$\alpha_{\text {reg }}$ is the ratio of the regulating and the secondary winding. $\lambda_{\text {reg }}$ is the copper filling factor of the regulating winding in $\%$. $U_{\text {reg }}$ is the voltage drop on the regulating winding in $k V$. $J_{\text {reg }}$ is the current density in the regulating winding in $\mathrm{A} / \mathrm{mm}^{2}$. $\varepsilon_{\text {main }}$ is the main winding regulating range in $\%$.

\section{7) Simple Limitations}

It is necessary to take into consideration a lot of requirements, like simple limitation of physical parameters such as saturation point of the magnetization curve

$$
B \leq B_{\max },
$$

overheating with the current density limitations

$$
J_{i} \leq J_{i_{\max }}
$$

and minimum distance for the main gap, the optimization algorithm can grow the short-circuit impedance, with higher main gap selection

$$
g \geq g_{\min }
$$

\section{8) Short-Circuit Impedance}

This is the weak point of the model described in [2], [7] for core-form transformers. The well-known analytical formula for the short circuit impedance calculation, which you can find in a wide range of transformer books [2], [15] and [16]

$$
Z=\frac{2 \pi^{2} \cdot \mu_{0} \cdot f \cdot P_{p h}}{U_{T}^{2} \cdot(h+s)}\left(\frac{R_{s} t_{s}}{3}+\frac{R_{p} t_{p}}{3}+R_{m} t_{m}\right),
$$

where $U_{T}$ is the turn voltage, all other parameters are known. However, there are several difficulties, why we use this formula to check the optimization result:

(I) This is a polynomial formula, so it is used for inequality constant only, as we seen it; The short-circuit impedance is a prescribed value, so we have to use it in at least two inequalities in $\pm 3 \%$ region, so it can give a solution which corresponds to the standard;

(II) The greater than inequality is the critical one, because the lesser impedance can reduce the transformer cost, so the equation does not constrain the cost function, like [2], [7] concluded.

So there is a need in constraint function for the optimization model in the simplified form $(A, B, C, D$ terms mean a general monomial expression)

$$
\frac{c_{1} \cdot Z}{A+B+C+D} \leq 1
$$

However, we can use only the pair of this formula to calculate the short-circuit impedance

$$
\frac{c_{1} \cdot(A+B+C+D)}{Z} \leq 1 .
$$

The GP module cannot handle the constraint expressions in the next form,

$$
\frac{c_{1}}{A+B} \leq D \text {. }
$$

Utilizing only one of the given two constraint functions like [2], [7] causes higher short-circuit impedance than the given short-circuit impedance value, so the optimized transformer 
has lower short-circuit impedance value. So we left these short-circuit impedance constraints from our equation system. To solve the problem of the short-circuit impedance calculation, we decided to introduce a heuristic solution based on the successive over-relaxation of the equation system. The basis of this solution is a shape factor, which describes the ratio of the primary and secondary winding radial widths, this factor derived from one monomial term of the short-circuit impedance.

The program iterates this factor. As a result of the first run, we get an optimized geometry, and we compare the calculated short-circuit impedance to the given. Usually the calculated short-circuit impedance lower than the given one. We slightly modify the geometry with an empirical shape factor defined with a monomial function in order to increase the calculated short-circuit impedance. The next run of the optimization, will find a new optimal geometry with the modified shape factor, and a higher short-circuit impedance. This procedure repeated till the calculated short-circuit impedance becomes close to the given one.

\section{Equality Constraints}

One of the most important constraint functions to describe the exact nominal phase power of the transformer winding

$$
P_{p h}=4.44 \cdot \eta_{c} \cdot \eta_{s} \cdot f \cdot \alpha \cdot h \cdot j_{s}^{2} \cdot B \cdot t_{s} \cdot R_{c}{ }^{2} .
$$

The abstract shape factor (fs):

$$
\frac{Z}{Z_{c}}=R_{s} \frac{t_{s}}{f_{s}},
$$

where, $Z_{c}$ is the aimed short-circuit impedance value. This empirical formula derived from one monomial term of (22).

\section{PRACTICAL EXAMPLE}

It is supposed that we have a calculation for a three-phase 33 MVA transformer with YNd11 connection. The secondary voltage is $34.5 \mathrm{kV}$ the primary is $161 \mathrm{kV}$, with a $\pm 10 \%$ regulating range. $11.2 \%$ is the short circuit impedance target. The capitalized loss prices $A=1400.0 € / \mathrm{kW}, B=4000 € / \mathrm{kW}$. The transformer has a 3 phases, 3 legged cores, the applied core material is a $\mathrm{M} 1 \mathrm{H}$ grade electrical steel. The price of the steel is $3.0 € / \mathrm{kg}$, the allowed maximum magnetic flux density in the transformer's core is $B_{\max }=1.65 \mathrm{~T}$. The core filling factor is set to $89 \%$. From the assumed winding types, the copper filling factor selected to $55 \%$ for the primary and the secondary windings, and $70 \%$ for the regulating one. The insulated copper price for the secondary winding is $10 € / \mathrm{kg}$, which a good estimation in the case of CTC conductors with helical winding arrangement and $9.0 € / \mathrm{kg}$ is assumed for primary disc and regulating winding. The main gap minimum has been chosen to $45 \mathrm{~mm}$, from the insulation levels according to [17], $B I L=650 \mathrm{kV}$ and $A C=275 \mathrm{kV}$. We set the core-secondary distance to 20 , and the distance between the two phase windings to 150 considering the insulation levels [15], [16] and [18].
TABLE I

\begin{tabular}{|c|c|c|c|c|}
\hline \multirow{2}{*}{\multicolumn{2}{|c|}{$\begin{array}{l}\text { Quantity } \\
\text { Method }\end{array}$}} & \multirow[t]{2}{*}{ Dimension } & \multicolumn{2}{|l|}{ Result } \\
\hline & & & I & II \\
\hline \multicolumn{2}{|l|}{ Turn Voltage } & V & 98.75 & 129.5 \\
\hline \multicolumn{2}{|c|}{ Induction in the column } & $\mathrm{T}$ & 1.65 & 1.65 \\
\hline \multicolumn{2}{|l|}{ Core diameter } & $\mathrm{mm}$ & 622.0 & 700 \\
\hline \multicolumn{2}{|l|}{ Core Mass } & $\mathrm{t}$ & 23.0 & 29.5 \\
\hline \multicolumn{2}{|l|}{ No-load loss } & $\mathrm{kW}$ & 22.2 & 28.5 \\
\hline \multicolumn{2}{|l|}{ Load loss } & $\mathrm{kW}$ & 129.95 & 76 \\
\hline \multicolumn{2}{|c|}{ Short-Circuit Impedance } & $\%$ & 11.39 & 6 \\
\hline \multirow{3}{*}{ Winding thickness } & secondary & $\mathrm{mm}$ & 100.0 & 62 \\
\hline & primary & $\mathrm{mm}$ & 95.0 & 78 \\
\hline & regulating & $\mathrm{mm}$ & 11.0 & 11 \\
\hline \multirow{3}{*}{ Winding height } & secondary & $\mathrm{mm}$ & 1033.0 & 1058 \\
\hline & primary & $\mathrm{mm}$ & 992.0 & 1015 \\
\hline & regulating & $\mathrm{mm}$ & 579.0 & 592 \\
\hline \multirow{3}{*}{ Current density } & secondary & $\mathrm{A} / \mathrm{mm}^{2}$ & 1.96 & 2.44 \\
\hline & primary & $\mathrm{A} / \mathrm{mm}^{2}$ & 2.07 & 1.96 \\
\hline & regulating & $\mathrm{A} / \mathrm{mm}^{2}$ & 2.32 & 2.03 \\
\hline \multirow{3}{*}{ Mean diameter } & secondary & $\mathrm{mm}$ & 756.0 & 796 \\
\hline & primary & $\mathrm{mm}$ & 1040.0 & 1025 \\
\hline & regulating & $\mathrm{mm}$ & 1295.0 & 1262 \\
\hline
\end{tabular}

RESULTS OF PRACTICAL EXAMPLE CALCULATION

The results of the calculation are in Table I. In turn, the Method I shows the results of the new algorithm, Method II shows the solution of the GP presented in[2], [7]. The Method II as it is shown calculates inadequate short-circuit impedance and the active-part design contains taller windings as well as core diameter is wider than results provided by the new method. The dimensions that we get with the new solution technique correspond to the designer's calculation.

\section{EXAMPLE - THEORETICAL CALCULATION}

A theoretical calculation is made based on the well-known function, which provides maximum efficiency for the transformer if the load loss equals with loss without load. This statement is true only in the case when the capitalization prizes are extremely high and the manufacturing prices are not considerable. It is important to note that the objective function simplified in this case to the following form:

$$
C=A P_{n l l}+B P_{l l} .
$$

The load loss and no load loss capitalization prices are set to $10000[€ / \mathrm{kg}]$ and the material price are reduced to $0.1[€ / \mathrm{kg}]$. All of the other technological parameters are taken from the practical example (previous chapter). So we expect that the core loss and load loss ratio near to $1: 1$, not exactly because of the applied factors, which we used to take into consideration the stray losses in the other construction parts of the transformer. 
TABLE II

RESULTS OF THE THEORETICAL EXAMPLE CALCULATION

\begin{tabular}{|c|c|c|c|}
\hline \multicolumn{2}{|l|}{ Quantity } & Dimension & Result \\
\hline \multicolumn{2}{|l|}{ Turn Voltage } & V & 103.5 \\
\hline \multicolumn{2}{|c|}{ Induction in the column } & $\mathrm{T}$ & 1.04 \\
\hline \multicolumn{2}{|l|}{ Core diameter } & $\mathrm{mm}$ & 800.0 \\
\hline \multicolumn{2}{|l|}{ Core Mass } & $\mathrm{t}$ & 62.4 \\
\hline \multicolumn{2}{|l|}{ No-load loss } & $\mathrm{kW}$ & 19.2 \\
\hline \multicolumn{2}{|l|}{ Load loss } & $\mathrm{kW}$ & 21.5 \\
\hline \multicolumn{2}{|c|}{ Short-Circuit Impedance } & $\%$ & 11.16 \\
\hline \multirow{3}{*}{ Winding thickness } & secondary & $\mathrm{mm}$ & 178.0 \\
\hline & primary & $\mathrm{mm}$ & 241.0 \\
\hline & regulating & $\mathrm{mm}$ & 11.0 \\
\hline \multirow{3}{*}{ Winding height } & secondary & $\mathrm{mm}$ & 2452.0 \\
\hline & primary & $\mathrm{mm}$ & 2354.0 \\
\hline & regulating & $\mathrm{mm}$ & 1447.0 \\
\hline \multirow{3}{*}{ Current density } & secondary & $\mathrm{A} / \mathrm{mm}^{2}$ & 0.41 \\
\hline & primary & $\mathrm{A} / \mathrm{mm}^{2}$ & 0.31 \\
\hline & regulating & $\mathrm{A} / \mathrm{mm}^{2}$ & 1.47 \\
\hline \multirow{3}{*}{ Mean diameter } & secondary & $\mathrm{mm}$ & 1011.0 \\
\hline & primary & $\mathrm{mm}$ & 1519.0 \\
\hline & regulating & $\mathrm{mm}$ & 1919.0 \\
\hline
\end{tabular}

The results can be seen in Table II. The diameter of the core and the winding dimension are very high because of the abnormal cost parameters, nevertheless this model demonstrates that the solution of this optimization method corresponds to the theory in this extreme case.

\section{CONCLUSIONS}

The presented transformer optimization model can help the designer to make a competitive solution in short time for a quotation. The introduced calculation examples require only few seconds to compare the solutions for a full quotation. In a standard way it takes more than one day. This paper showed that the geometric programming model, which introduced in [2], [7] and effectively used for shell type transformer optimization [5]. The previous method is not able to take the short-circuit impedance of the transformer into consideration. In this paper, a new heuristic method is introduced by sample calculations. The newly developed calculation method eliminates the weakness of the previous model by the correction of the short-circuit inductance calculation [2], [7].

\section{REFERENCES}

[1] G. Újházy, "Design of winding systems of power transformers", Elekrotechnika, pp. 450-459, November-December 1969.

[2] R.M. Del Vecchio, B. Poulin, P.T. Feghali, D.M. Shah, R. Ahuja, „Transformer Design Principles - With Applications to Core-Form Power Transformers", CRC Press, 2002.

[3] G. Újházy, "A transzformátortervezés gépi programozásának kérdései", Elekrotechnika, pp. 355-363, September 1969. Academic, 2003.

[4] O.W. Andersen, „Optimized Design of Electric Power Equipment”, IEEE Computer Applications in Power, Vol. 4, January 1991. http://dx.doi.org/10.1109/67.65030

[5] R.A. Jabr, „Application of Geometric Programming to Transformer Design", IEEE Transactions on Magnetics, 2005 November. http://dx.doi.org/10.1109/TMAG.2005.856921

[6] P.S. Georgialakis, „Spotlight On Modern Transformer Design”, Springer, 2009. http://dx.doi.org/10.1007/978-1-84882-667-0

[7] M.A. Masood, R.A. Jabbar, M.A.S. Masoum, M. Junaid, és M. Ammar, „An innovative technique for design optimization of core type 3-phase distribution transformer using mathematica", Global J. Technology \& Optimization (GJTO), vol 3, 2012.

[8] E.I. Amoiralis, P.S. Georgilakis, M.A. Tsili, A.G. Kladas, és A.T. Souflaris, „Complete Software Package for Transformer Design Optimization and Economic Evaluation Analysis", Materials Science Forum, vol 670, o 535-546, Dec. 2010.

[9] R.J.Duffin, E.L.Peterson, C.Zener, "Geometric Programming", Wiley, NY, 1967.

[10] S. Boyd, S.-J. Kim, L. Vandenberghe, és A. Hassibi, „A tutorial on geometric programming", Optimization and Engineering, vol 8, sz 1, o 67-127, ápr 2007.

[11] S.P. Boyd és L. Vandenberghe, Convex optimization. Cambridge, UK; New York: Cambridge University Press, 2004.

[12] M. Anderson, L. Vandenberghe, "CVXOPT-Python Software for Convex Optimization". http://cvxopt.org, 2012-2013.

[13] P.S. Georgilakis, „Environmental cost of distribution transformer losses", Applied Energy, vol 88, sz 9, o 3146-3155, szept 2011.

[14] S. Corhodzic and A. Kalam, ,Assessment of distribution transformers using loss capitalization formulae", Journal of Electrical and Electronics Engineering Australia, vol 20, sz 1, p. 43-48, 2000.

[15] Károly Karsai, Dénes Kerény, László Kiss, "Large power transformers", Elsevier, 1986

[16] S.V. Kulkarni, "Transformer Design and Practice", CRC Press, 2004.

[17] IEEE Loss Evaluation Guide for Power Transformers and Reactors, IEEE Std. C57. 120-1991, Sep. 1991.

[18] Zoltán Ádám Tamus, Complex diagnostics of insulating materials in industrial electrostatics, Journal of Electrostatics, Volume 67, Issues 2-3, May 2009, Pages 154-157, ISSN 0304-3886, http://dx.doi.org/10.1016/j.elstat.2009.01.054

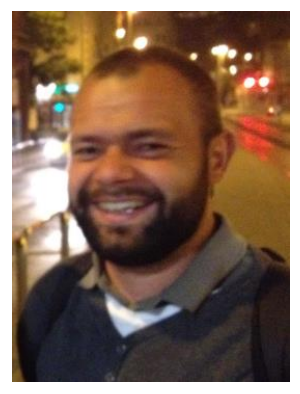

Tamás Orosz is received his MSc degree in Electrical Engineering from Budapest University of Technology and Economics (BUTE), Budapest, Hungary in 2012.

$\mathrm{He}$ is currently working as a software developer at High Voltage Solutions Ltd, and as a PhD student in Department of Power Electrical Engineering in BUTE, Hungary. His fields of research include power transformer optimization, numerical calculations of different parameters for power transformers.

Postal address: Budapest University of Technology and Economics, Department of Electric Power Engineering.

Address: Egry J. u. 18, H-1111 Budapest, Hungary

E-mail: orosz.tamas@vet.bme.hu

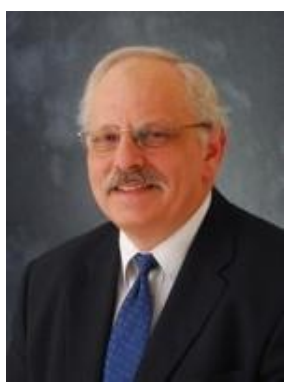

István Vajda (CSc, $\mathrm{PhD}, \mathrm{DSc}$, dr. habil) is from Obuda University, director of Institute of Automation, and as a part-time professor working with the Department of Electric Power Engineering, Budapest University of Technology and Economics. He is the leader of the SuperTech Laboratory. Fields of research: Large scale applications of superconductivity, Theory and design of electrical machines;, Engineering problem solving. Nonconventional energy conversion. Internationally acknowledged scientist and expert in large scale applications of superconductivity.

Postal address: 1034 Budapest, Bécsi út 94-96. C II. 217

E-mail: vajda.istvan@kvk.uni-obuda.hu 